\title{
A 33-year-old male with a strenuous occupation and gradual inability to lift, pull, and push heavy weights while working
}

\author{
Evangelos Perdikakis • Lampros Palladas • \\ Apostolos Karantanas
}

Received: 8 March 2011 /Revised: 6 June 2011 / Accepted: 4 July 2011 /Published online: 29 July 2011

(C) ISS 2011

\section{Question}

A 33-year-old male with a strenuous occupation, presented with a 6-week history of mild pain and right-sided arm weakness. His complaints had progressed slowly over the last few weeks and the patient revealed a gradual inability to lift, pull, and push heavy weights while working. The patient was initially on oral analgesics and lately on steroids (per os methylprednisolone, 4-mg tablets) for a period of 6 days prior to referral, but with only moderate improvement. The patient did not recall any recent or remote traumatic incidence. MR imaging of the cervical spine was normal and MR imaging study of the thoracic wall was performed subsequently upon request (Figs. 1-3).

The diagnosis can be found at doi:10.1007/s00256-011-1232-1. 


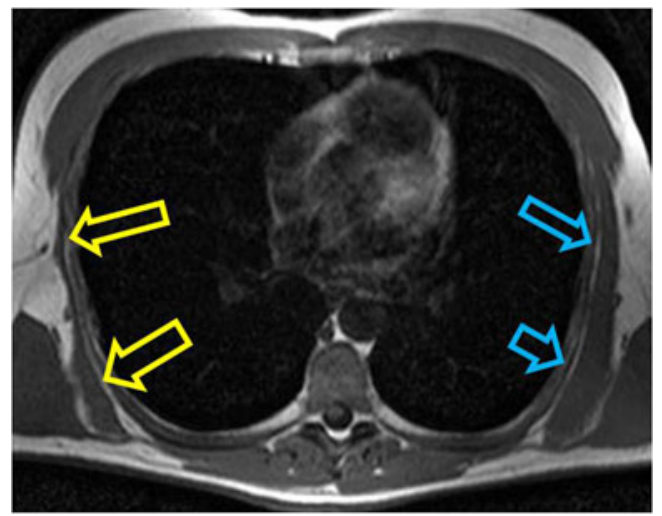

Fig. 1 The axial T1-w MR image shows atrophy of the right serratus anterior muscle (yellow arrows). The anterior portion of the muscle is particularly affected. Compare with the normal muscle mass of the left serratus anterior (blue arrows)

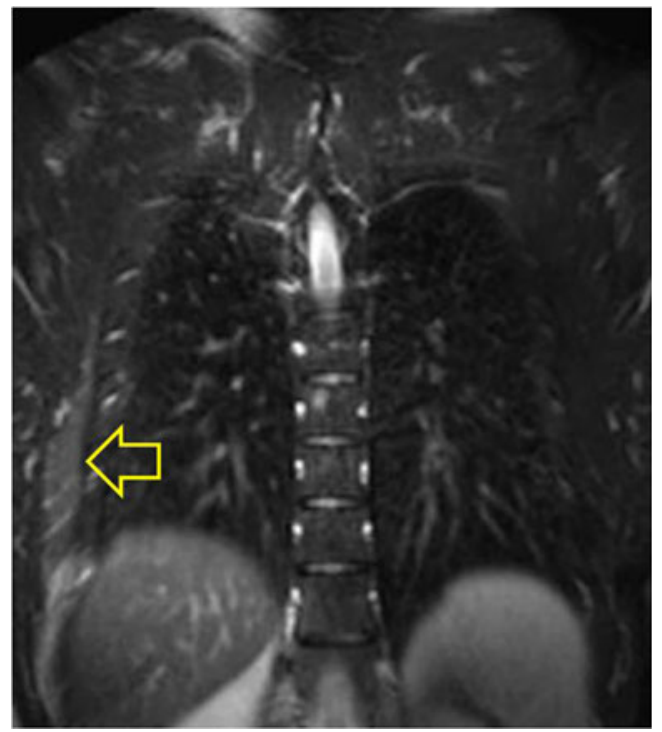

Fig. 2 The coronal fat-suppressed T2-w MR image demonstrates increased signal (arrow) of the anterior portion of the right serratus anterior muscle, a finding in keeping with subtle muscle edema
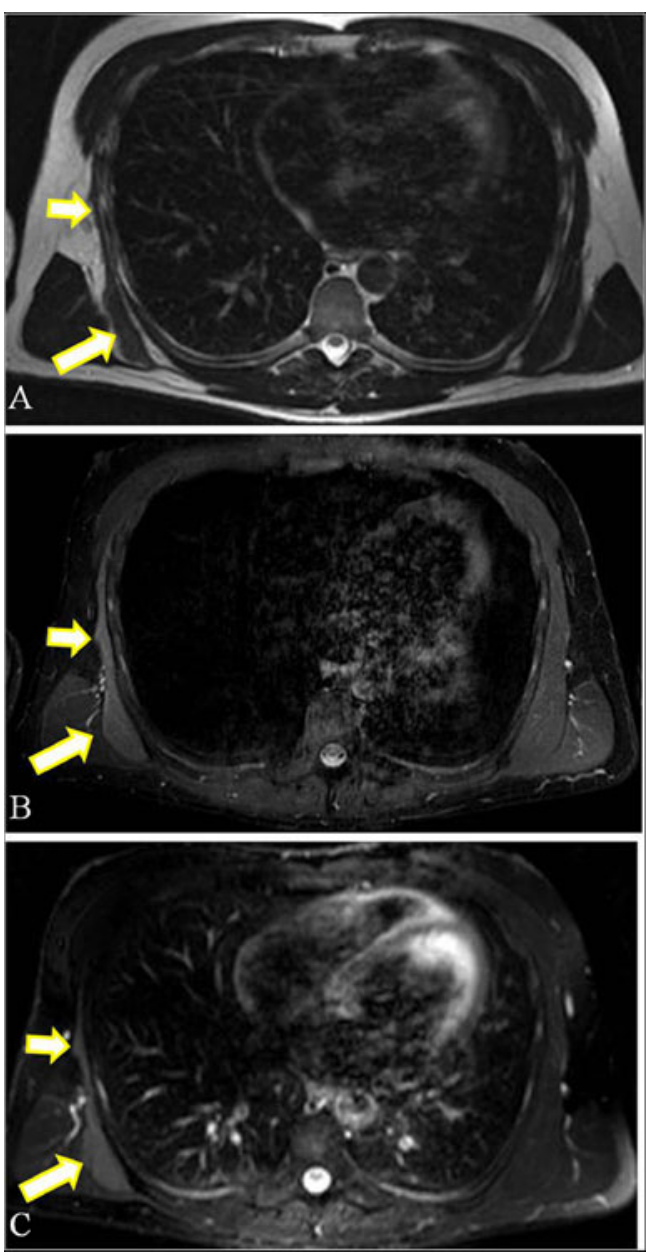

Fig. 3 The a axial T2-w TSE, b STIR and $\mathbf{c}$ fat-suppressed T2-w TSE MR images show atrophy and increased signal within the right serratus anterior muscle (arrows). The STIR image shows the edema best because of the heterogeneous fat suppression on a fat-suppressed T2-w TSE image 